\title{
SCANNING TUNNELS WITH TWO VERY HIGH-RESOLUTION LASER DEVICES AND A STACKER
}

\author{
P. Foucher ${ }^{1}$, P. Charbonnier ${ }^{1, *}$ T. Noël ${ }^{2}$, Y. Fosse ${ }^{2}$, J.-F. Hébert ${ }^{3}$ \\ ${ }^{1}$ Cerema, Project-Team ENDSUM, 67100 Strasbourg, France - (philippe.foucher, pierre.charbonnier)@ cerema.fr \\ ${ }^{2}$ Cerema, Centre d'Etudes et de Construction de Prototypes, 76120 Le Grand Quevilly, France - (thibaut.noel, yann.fosse)@ cerema.fr \\ ${ }^{3}$ Pavemetrics, Québec, QC G1R 5B1, Canada - jfhebert@pavemetrics.com
}

\section{Technical Commission II}

KEY WORDS: Tunnel inspection, Laser Scanning, High-resolution, Mobile acquisition, Defect detection.

\begin{abstract}
:
In this paper, we analyse the potentialities of the LCMS (Laser Crack Measurement System) sensors for tunnel inspection. Two of these sensors have been integrated into a flexible prototype developed by Cerema, allowing data from the entire tunnel to be recorded dynamically in a few passes. Thanks to this system, we were able to acquire, in a limited time, range and intensity images of the complete vaults of four tunnel tubes, with high spatial resolution, during experimental campaigns. We present image processing methods to take advantage of this type of image. These approaches initially involve improving data visualization and reconstructing a local 3D model. We propose some preliminary approaches to correct the distance image in order to better highlight local variations in depth. These data are currently being deployed for learning neural networks for defect detection.
\end{abstract}

\section{INTRODUCTION}

In this contribution, we introduce a mobile high-resolution $3 \mathrm{D}$ data acquisition prototype, composed of two very highresolution sensors, allowing the entire tunnel vault to be surveyed in a small number of passages.

This work is part of a project that aims to implement visual inspection tools capable of automatically detecting disorders (cracks, material loss) from 3D data. There are currently relatively few automated inspection systems dedicated to tunnels. Most of them combine various imaging modalities (visible, infrared) with laser acquisition (Loupos et al., 2014). The latter is mostly used for large-scale diagnoses of the tunnel geometry. However, local depth variations are characteristics specific to certain defects and we believe that this information may be useful to facilitate their detection. This clearly requires obtaining data with sufficient resolution to highlight these local depth changes on tunnel walls and vaults. In the context of a research and development partnership, we have chosen to experiment with sensors from the Canadian company Pavemetrics, which provide a spatial resolution of the order of a millimeter and a sub-millimetric depth resolution.

The acquisition of data in tunnels poses a number of technical and practical difficulties and compromises often have to be made. The sometimes variable geometry of tunnels, and the presence of equipment, which can be large, require that the deployed devices be as adaptable as possible. Tunnel closure periods are rare, for economic reasons, and they should be kept as short as possible. This means that data acquisition must be made possible in a context of sometimes very constraining coactivity. Rather than reducing the time spent scanning the tunnels, which would have an obvious negative impact on the resolution of the acquired data, it may be interesting to reduce the adjustment times between passages. We have chosen to develop a relatively simple system, which is both rigid enough to ensure

${ }^{*}$ Corresponding author high quality acquisitions and flexible enough to be configurable in a reasonable amount of time.

In this paper, we propose a description of this prototype system, how we implemented it in real world conditions during experimental campaigns in tunnels, and the potentialities offered in terms of visualization, 3D reconstruction, and image analysis.

The rest of the paper is organized as follows. In Section 2, we introduce the sensors used and how they were embedded in a prototype vehicle. In Section 3, we describe the acquisition campaigns we conducted using this prototype. In Section 4, we show the data obtained and illustrate their potentialities. Section 5 concludes the paper and provides an opening to work perspectives.

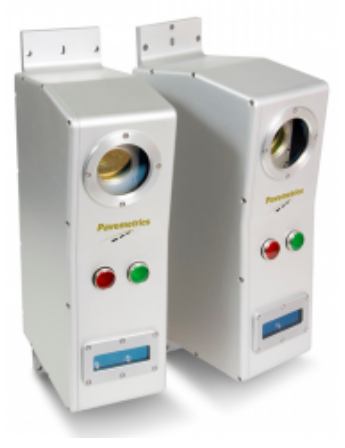

Figure 1. Pavemetric's LCMS-2 sensors.

\section{MATERIALS}

In this section, we introduce the protoype we developed to inspect tunnels using LCMS sensors.

\subsection{LCMS sensor}

Pavemetrics has developed the Laser Crack Measurement System (LCMS), initially dedicated to road inspection. Embed- 
ded on a carrier vehicle, it allows 3D data to be acquired at high speed and high resolution. In its most recent version, the LCMS-2, shown in figure 1, 28,000 profiles with more than 4,000 points spaced one $\mathrm{mm}$ apart can be acquired per second, with a depth resolution in the order of one tenth of a millimeter. The arrival of the LCMS sensors on the market has provided an industrial solution to the problem of detecting pavement defects, a topic that has generated significant literature over the past three decades. We refer the reader to recent surveys, e.g.(Radopoulou, Brilakis, 2016, Mohan, Poobal, 2018). Today, LCMS is a leading product, with more than 200 systems sold worldwide. Cerema uses these sensors, embedded in its Aigle3D inspection vehicle (Cerema, 2019): every year, about 23,000 km of pavement are inspected in France.

In recent years, these systems have also been implemented for tunnels, under the name of Laser Tunnel Surveying System or LTSS, see (Gavilan et al., 2013), (Laurent et al., 2014). LTSSbased inspection vehicles incorporate several sensors arranged in a circular arc to cover half of the vault or even the entire vault. These systems are calibrated and allow a 3D model of the tunnel to be reconstructed. The positions and orientations of the sensors must be modified according to the geometric characteristics of each tunnel, which may require a significant installation time on site. On the other hand, the number of passages in the tunnel is limited and post-processing is easier. In the first phase of our project, the priority is not the overall 3D reconstruction of the tunnel but the learning of local defects, and we have therefore chosen to develop a simplified system composed only of two LCMS sensors, easily orientable and repositionable. The installation time on site is greatly reduced, even if it obviously requires more passages to cover the entire vault.

LCMS sensors consist of a laser transmitter and a line camera. The deformations of a laser line, projected on the surface of interest, are observed using a high frequency digital camera. Trigonometric rules are used to calculate the distance between the point on the target surface and the camera. The resulting profile, which includes 2080 pixels for the LCMS- 1 sensor, is measured both in intensity and depth. The lateral resolution is $1 \mathrm{~mm}$.

Data recording is triggered at a fixed frequency and the profiles are located along the progression path of the vehicle, using a high-precision mechanical odometer fixed on the axis of a wheel. More specifically, it is a 5000 point incremental encoder, used in 4 quadrants, which means a 20000 point acquisition for a $\varnothing 670 \mathrm{~mm}$ wheel, or a $2014 \mathrm{~mm}$ development. The odometry resolution is therefore in the order of the $10^{\text {th }}$ of $\mathrm{mm}$ and the triggering can be millimetric. In practice, one profile is acquired every $2 \mathrm{~mm}$, the acquisition speed being about $8 \mathrm{~km} / \mathrm{h}$.

The sensors are equipped with inertial measurement units (IMUs) that capture accelerometric and inclinometric data at a frequency of $150 \mathrm{~Hz}$.

\subsection{MALT Prototype}

The prototype we have implemented is called MALT (for Mobile Acquisitions with Lasers in Tunnels). It was designed and built in just a few weeks by Cerema's prototype construction facilities (CECP) in Rouen.

It consists of two LCMS-1 sensors attached to a steerable platform, itself attached to a stacker (see figure 2). The relative orientation between the two sensors, which is adjustable, was fixed to ensure a certain overlap between their fields of view, and remained the same throughout the acquisition campaigns.

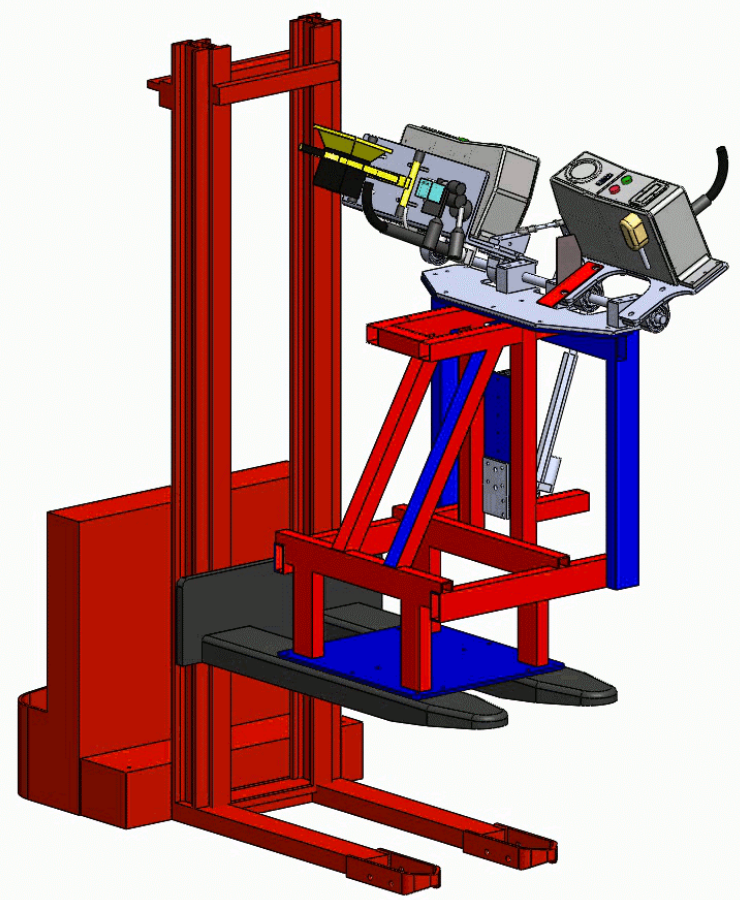

Figure 2. Prototype design: view of the stacker equipped with the mobile platform supporting two interdependent LCMS sensors.

The whole system is mounted on a truck. With this prototype, several passages ( 6 to 7 , in practice, depending on the geometry of the structure and the equipment present) are required to inspect the entire tunnel tube. The height and orientation of the sensors is adjusted before each passage.

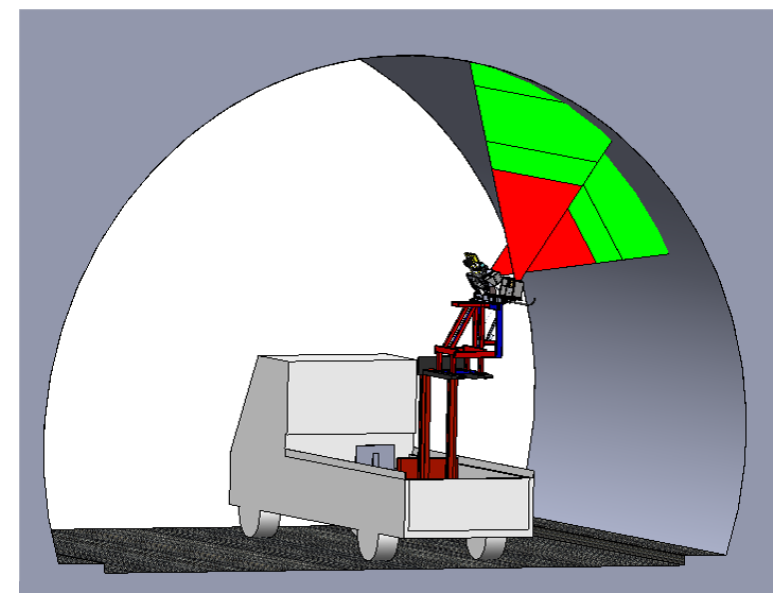

Figure 3. The field of view constraint. The area of correct focus is shown in green.

The knowledge of the geometry of the system laser - projected point - camera is essential to make correct acquisitions. The distance between the sensor and the target surface must be calculated so that the laser line remains within the camera's field of view (shown in green on figure 3). While in the case of pavement inspection, the sensors are positioned vertically and their height above the ground varies little, in the case of tunnels, several position and orientation adjustments are necessary and this 
must be done while respecting the field of view constraint.

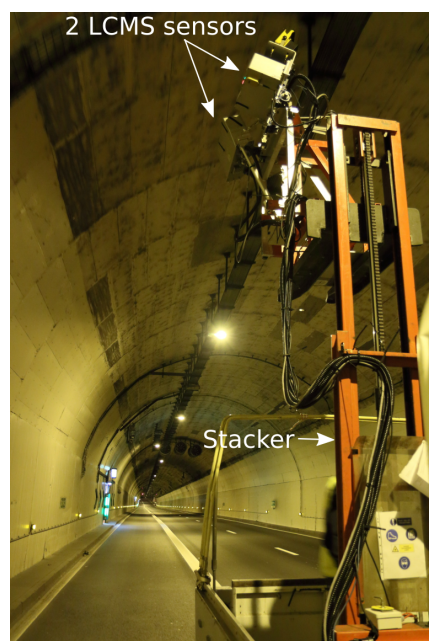

Figure 4. MALT Mobile prototype deployed in a tunnel.

To best adjust the position and orientation of the sensors, one can physically vary three parameters. The height required to acquire the desired area of interest on the vault is reached by means of the stacker, by means of an electric control system. The target height is checked using a laser rangefinder. The orientation of the plate supporting the sensors is controlled by means of a actuator and the angle is measured by means of a protractor. Finally, a side-looking lidar is attached to the front of the truck in order to record the distance between the tunnel wall and the vehicle, that must remain as constant as possible to respect the field of view constraint. A system including a side-

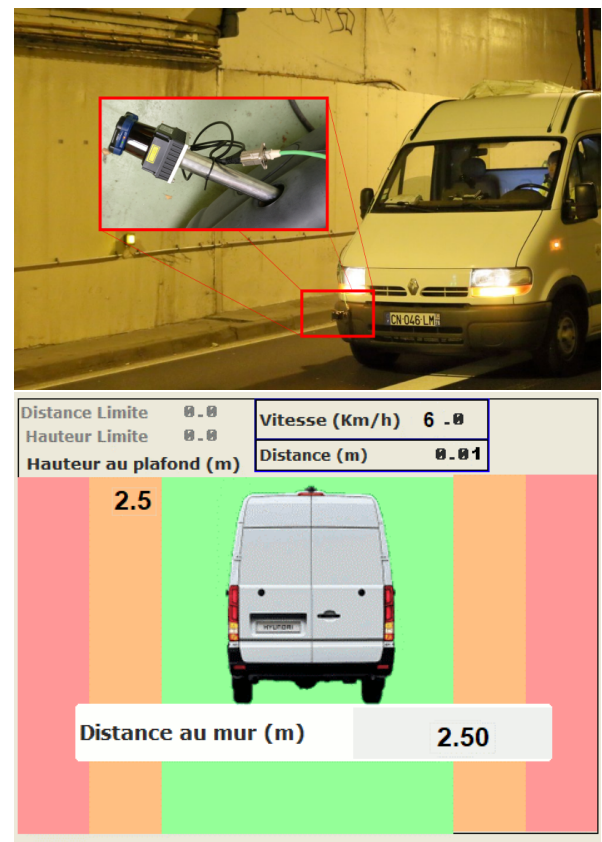

Figure 5. Positioning of the front LIDAR (top) and interface for checking the distance between the vehicle and the tunnel walls (bottom).

looking lidar, mounted at the front of the vehicle and a software interface, has been specially developed so that the vehicle driver can permanently visualize on a screen the lateral position of the vehicle and adjust its trajectory accordingly (see figure 5). To simplify the installation of the sensors, a preliminary study is carried out on the basis of the cross-sectional profile of the tunnel, as shown in figure 6 . The number of passages required in the tunnel, as well as the height and angle adjustments, are thus pre-calculated. In this way, the initial installation on site takes less than half an hour, and changes in settings between passages only take a few minutes.

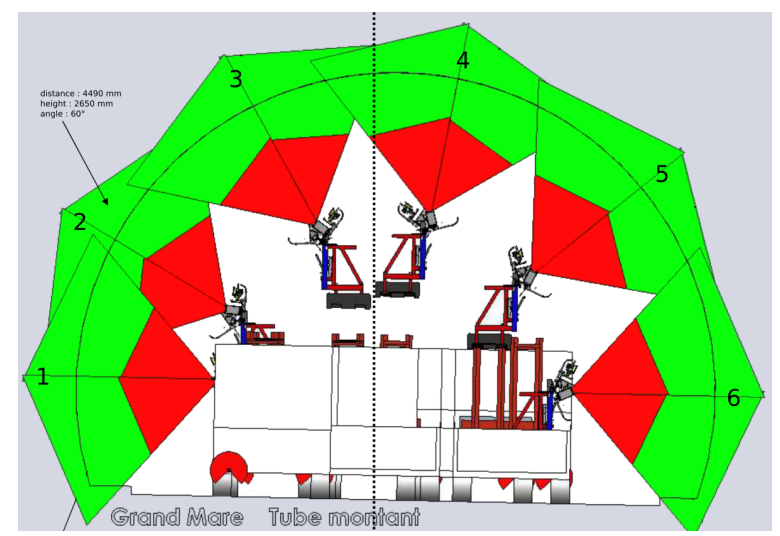

Figure 6. Distance, height and angle parameters pre-computed for every passage in a tunnel.

\section{EXPERIMENTS}

In this section, we describe the three experimental campaigns that were carried out using the MALT prototype.

\subsection{Experimental sites}

Three acquisition campaigns were carried out in motorway tunnels near Paris (France), as shown on figure 7: the Saint-Cloud tunnel (832 $\mathrm{m}$ - south tube), the Chennevière covered trench near Jouars-Pontchartrain ( $450 \mathrm{~m}$ - south tube) and the "GrandMare tunnel" in Rouen (1500 m - 2 tubes). These campaigns were done at night, between $10 \mathrm{pm}$ and $4 \mathrm{am}$, as part of tunnel maintenance periods. The speed of the MALT vehicle, limited to $8 \mathrm{~km} / \mathrm{h}$, was actually $6 \mathrm{~km} / \mathrm{h}$, which allowed the desired resolution of $2 \times 1 \mathrm{~mm}$ to be achieved.

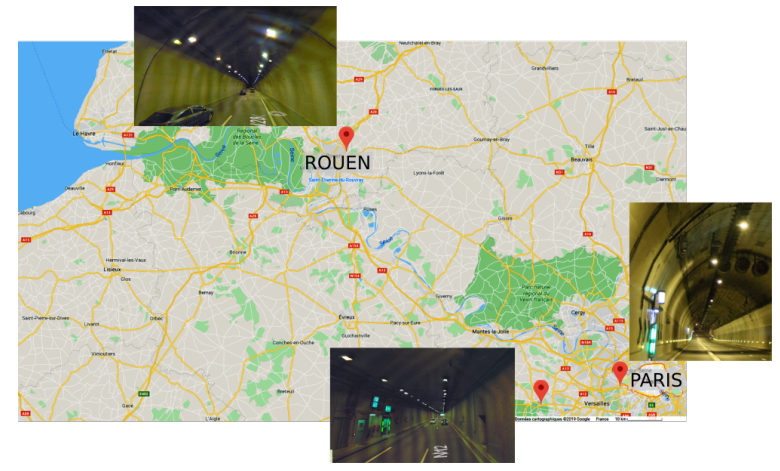

Figure 7. Localization of the surveyed tunnels

The St-Cloud and Grand-Mare tunnels have a tubular section, while the covered trench in Chennevière has a rectangular section. All three structures are coated with concrete, excepted Chennevière, which is partly covered with a metal coating.

\subsection{Data recording}

All acquisitions could be completed within the allotted time: one night of closure in St-Cloud and one in Chennevière, two nights in the Grand-Mare tunnel. 
At each position, the pair of LCMS sensors used on MALT produces two pairs of images (one intensity image and one range image) comprising 500 profiles, i.e. covering an area of $1 \times 2 \mathrm{~m}$, with a slight overlap. These images are grouped in a compressed file format (with a .fis extension). Note that there is no overlap between successive images of the same sensor.

The survey of the southern tube of Saint-Cloud tunnel needed 6 passages and produced about 830 files per passage and per sensor. In Chennevière, 6 passages were made in the south tube, providing an average of 380 files per passage and per sensor (the metal-covered sections were not surveyed). Finally, in Rouen Grand-Mare, 7 passages were necessary in each tube. While the presence of works has had little impact on acquisitions in the west tube, this co-activity precluded the investigation of a part of the east tube (near the entrance). Finally, 1360 files were produced per passage and per sensor on average for each tube. In total, a volume of more than 26300 .fis files was produced per sensor.

\section{DATA PROCESSING}

In this section, we illustrate the uses that can be made of the intensity and range images from LCMS sensor in the context of tunnel inspection.

\subsection{Image visualization}

In our configuration, the images of the two sensors are shifted along to axes and oriented head-to-tail with respect to each other, as illustrated on figure 8. It is therefore necessary to coregister them in order to be able to view them correctly.

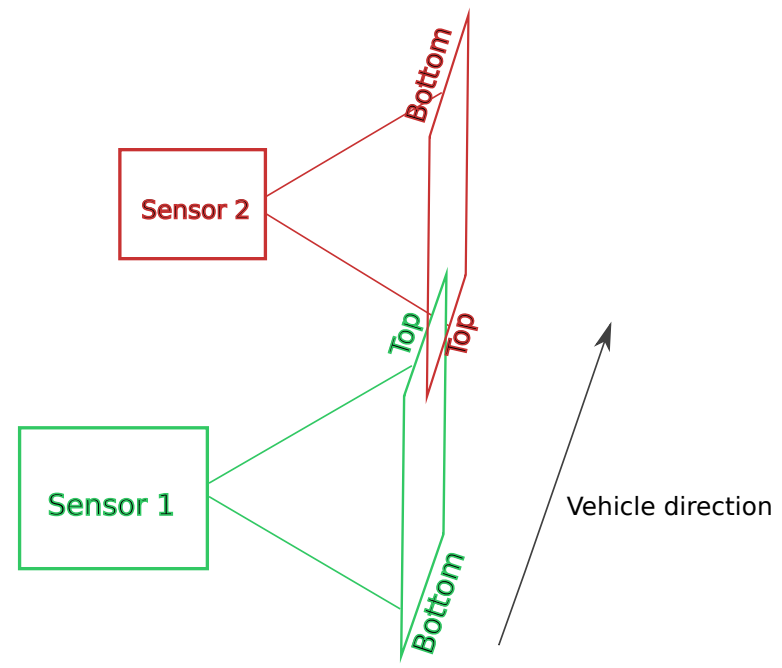

Figure 8. Relative orientation and position of the images taken by both sensors at each acquisition.

To do this, the first step is to determine the offset between the images. It is assumed that the common surface is locally flat (which seems reasonable, as the estimation is performed on a 150 pixel band on the corresponding borders of the images) and two translations are sought in order to superimpose the images. For this purpose, we use phase correlation, which exploits the properties of the Fourier transform to efficiently compute the translation vector. It is easy to show that the Fourier transform of the correlation of the images peaks at a position that corresponds to the coordinates of the displacement vector. As correlation is implanted by a product in the Fourier domain, the method is very fast. More details on phase correlation may be found in, e.g. (Szeliski, 2010, pp. 341-344), and a sub-pixelic implementation is described in (Foroosh et al., 2002).
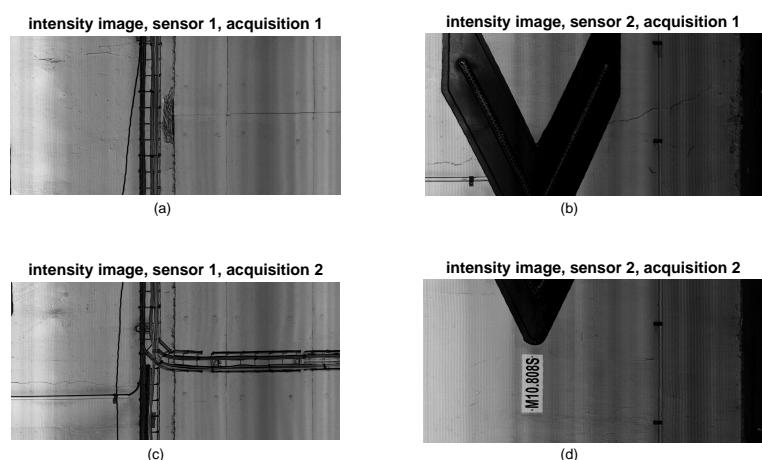

Figure 9. Images (a-b) and (c-d) represent two successive acquisitions. Image (c) must be flipped, shifted and merged with image (b) to form a continuous view of the tunnel wall.

The second step is to stitch the images together. To do this, they are superimposed and an alpha-blending technique is used to equalize their intensities. The same displacement vector is applied to the depth images as to the intensity ones.
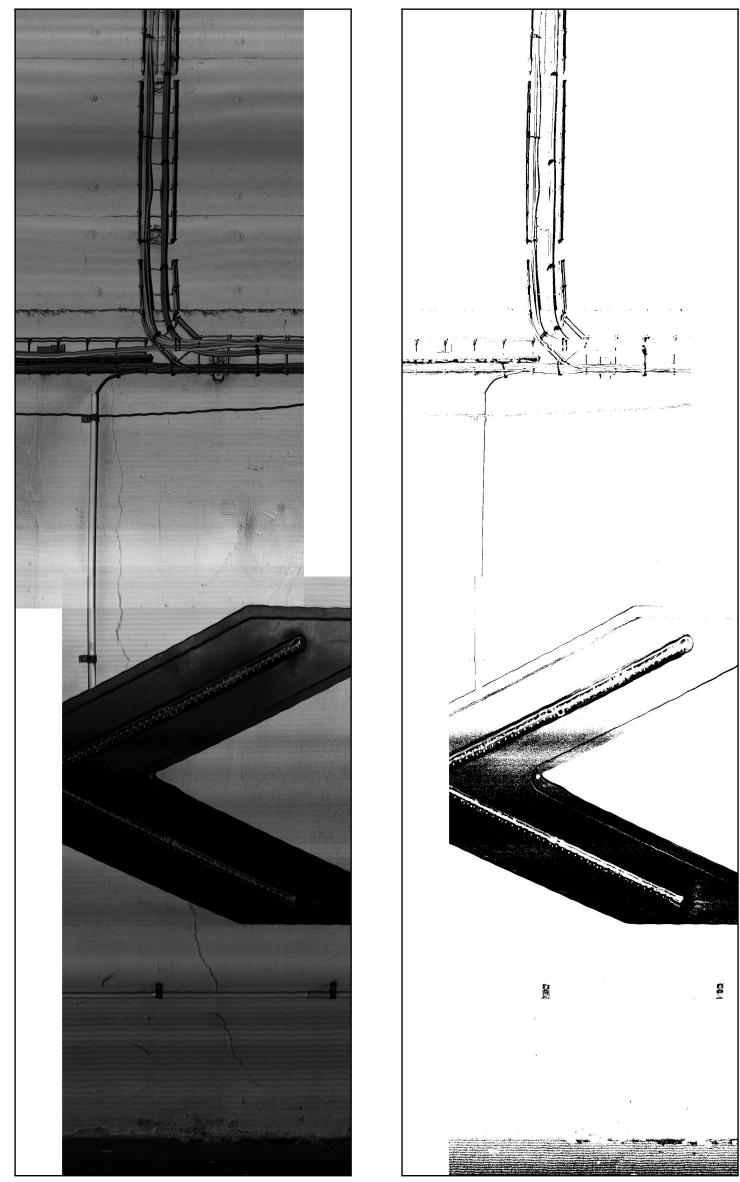

Figure 10. Stitching of the intensity (left) and depth (right) images of a tunnel wall. The translation vector was obtained from images (b) and (c) in figure 9.

An illustrative result (obtained from the acquisitions in figure 9), that shows strips of intensity and range covering an area 
from the pavement to the tunnel vault kidney, is shown in figure 10. Since successive acquisitions are disjointed, it is sufficient to concatenate the stitching strips to cover larger areas, as shown in figure 11.

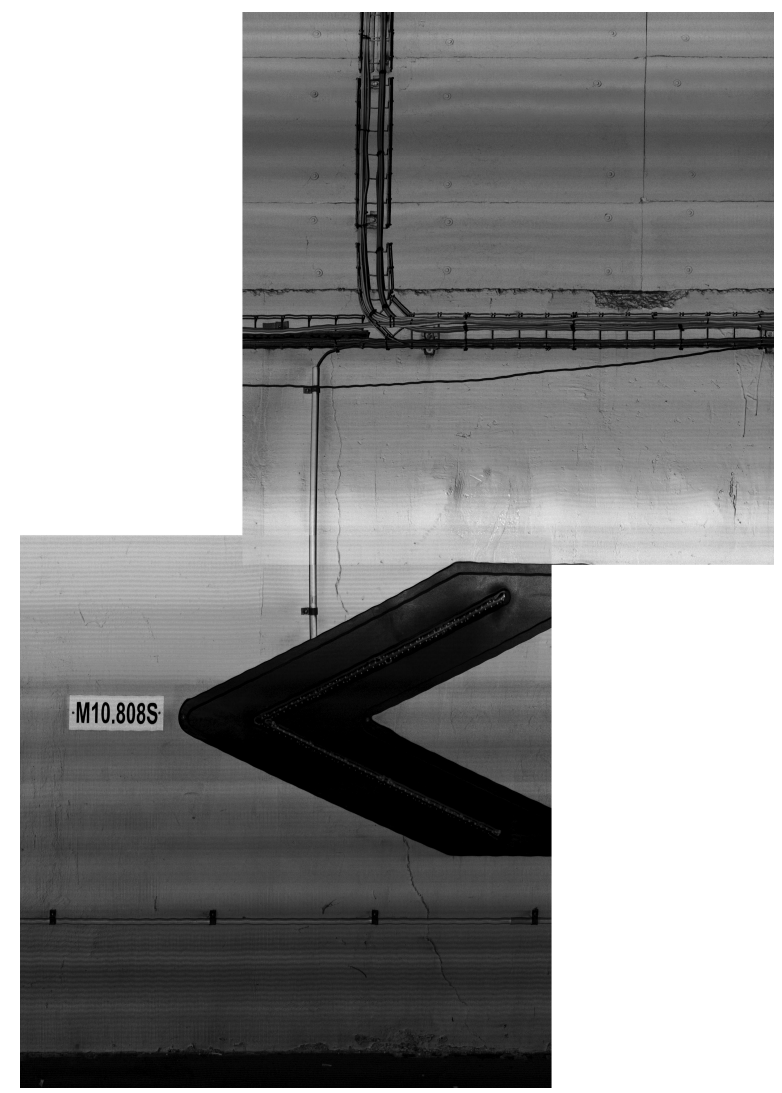

Figure 11. Image stitching (intensity) using two successive strips. A vertical crack is visible on either side of the signalling arrow, and a spall can be seen above the cable duct.

The images in figure 9 were acquired with a resolution of $1 \times 1 \mathrm{~mm}$ during a preliminary test, at a very low speed (about $4 \mathrm{~km} / \mathrm{h}$ ). In all subsequent acquisitions, the speed was about $8 \mathrm{~km} / \mathrm{h}$ in routine, so the native resolution of the images acquired by the sensors is $1 \times 2 \mathrm{~mm}$. To increase visual comfort during viewing, it is possible to restore the aspect ratio of 1 by upsampling images to a $1 \times 1 \mathrm{~mm}$ resolution using e.g. bi-cubic interpolation, even if it is advisable to perform image processing and analysis at native resolution.

Like all laser sensors, LCMS are sensitive to the photometric properties of the observed surfaces. For example, some very dark surfaces absorb the signal and the detection of the projected line cannot be done correctly. It may also happen that some areas of the image are outside the focus area. Finally, the signal can be diffracted or occluded. In all these cases, the invalid/outof-range values are indicated in the depth image by a very negative, constant value (-10000). These values must be filtered out because they overwhelm the dynamics of the images, as can be seen in the right-hand image in figure 10, where they appear in black. In addition, the general shape of the tunnel, which is often curved, also occupies an important part of the dynamics and can mask the details of the tunnel surface that we would like to highlight. To overcome these difficulties, it may be interesting to rectify the range images. This operation consists in calculating the difference between the measured points and a surface adjusted on them.
For pavement surfaces, for which the LCMS sensors have been designed, it is quite natural to fit a unique smooth reference surface. However, tunnels have much more complex surfaces. Their shape itself can include discontinuities, and the many pieces of equipment found in tunnels are outliers that can compromise the estimation. We are developing a method that automatically and robustly detects the presence of several connected components of sufficient size in profiles extracted perpendicular to the axis of the tunnel. Once this detection is performed, a robust surface fitting (using M-estimators (Huber, 1981, Tarel et al., 2002)) is performed on each of the corresponding parts of the image.

Finally, the rectified range is computed as the difference between the observed values and their values adjusted by the model. Invalid/out-of-range (-10000) data are given an arbitrary value. Pieces of equipment are detected by a simple thresholding. A preliminary result, obtained from image (a) in figure 9, is shown in figure 12 . The intensity image and the original depth image are shown on the upper part of the figure. On the range image is drawn the line 600 , which passes through a spall, and whose profile is shown below (in blue). On the profile, we show the cross-section of the two surfaces that have been robustly fitted on the left and right sides of the depth image. Below this graph is shown the rectified depth profile obtained from these smooth piecewise adjustments (the fitted surface are affine in the direction of the tunnel and of degree 4 perpendicular to it). The corresponding rectified depth image is shown at the bottom left of the figure. Note that to further improve the dynamics in the presence of the spall, which appears as a dark spot on the image, an exponential function was applied before the display. Our first experiments show that, to make visualization of defects easier for an operator, it may be interesting to modulate the coefficient of the exponential applied to the depth image in an interactive way to adapt it to the data.

\subsection{D model}

Based on the depth and image data contained in the fis files, it is possible to reconstruct local 3D representations of the structure. This requires calibrating the relative geometry of the sensors, which can be done using data from the IMUs and matching methods. The points can then be represented in a common local 3D frame, which can be attached to the sensors. The model is stored in a .las file. This model, an example of which is shown in 13, can be visualized using specialized software: its interactive manipulation often allows a better interpretation of the data (especially for elements such as cornices, cables, joints...).

In our experiment, we did not focus on the complete 3D reconstruction of the structure. Therefore, the 3D models have so far only been recalculated in strips, each one corresponding to a passage through the tunnel, with a particular position and orientation of the sensors. It would be possible to register all these bands, using techniques such as Iterative Closest Point, ICP (Besl, McKay, 1992), for example, and using the knowledge of the orientations and heights applied to the sensors during acquisitions. To avoid drift, control points identified by conventional topometric techniques should be used.

\subsection{Image analysis}

The example in figures 12 and 13 shows the importance of using $3 \mathrm{D}$ information in conjunction with intensity information to search for defects such as cracks or spalls on tunnel walls. Three other examples that support this conviction are shown 


\section{Intensity image}

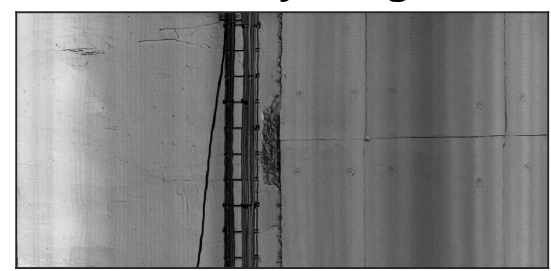

\section{Rectified range image}

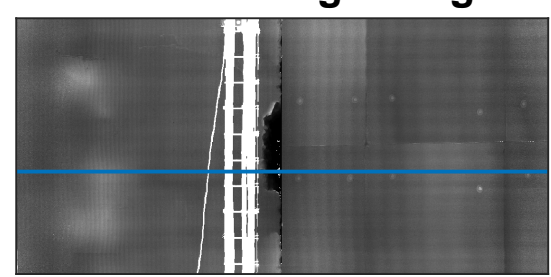

Range image

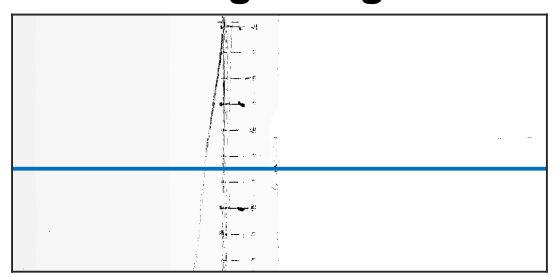

Range image profile

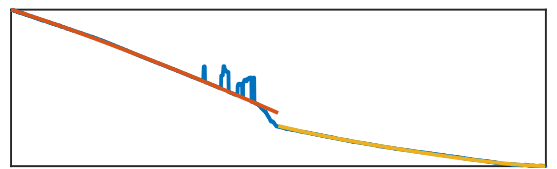

Rectified range image profile

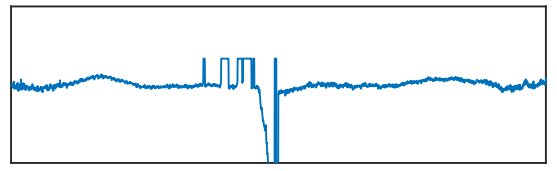

Figure 12. Range image rectification (see text). A piecewise smooth surface is adjusted on the range image (red and yellow curves on the profile). The spall is visible in the rectified range image and profile.

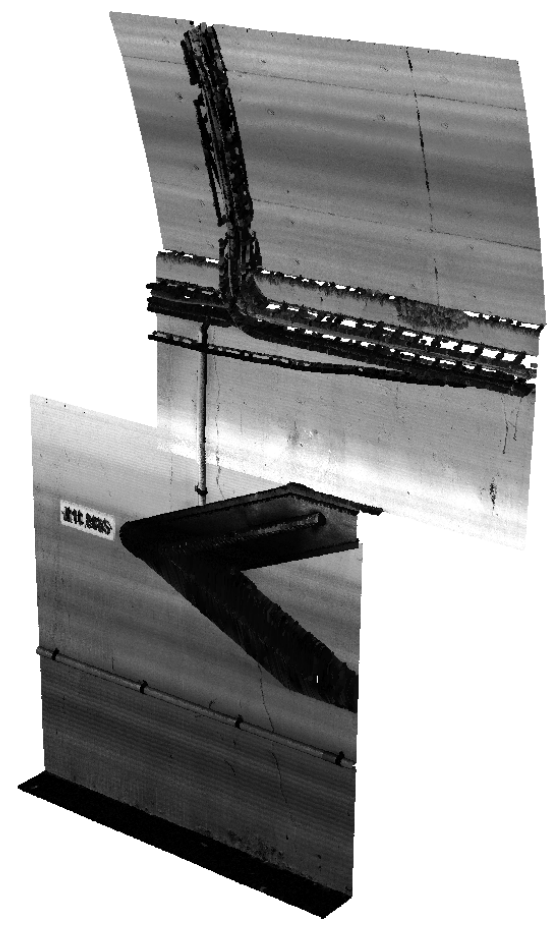

Figure 13. Local 3D model corresponding to the images in fig. 11. The points are colored using the intensity data.

in figure 14. The first one illustrates the possibility of detecting equipment, such as plates, or pipes, as well as out-of-range areas, from depth images. As such, equipment detection is not the primary focus of our study. However, these are elements that can interfere with defect detection. In addition, their identification and location may be an interesting secondary objective, in order to provide tunnel managers with inventory tools. In the second case, paint flakes, difficult to interpret on the intensity image, are highlighted in the range image. In the third image, a crack is clearly visible, both on the intensity image and on the depth image. The crack cuts three times the line along which the range profile is taken. The examination of the profile indicates that the crack manifests itself in peaks and valleys. In reality, peaks correspond to invalid values $(-10000)$, which are linked to occlusion phenomena. For visualization, it may be interesting to filter them, but for a pattern recognition algorithm, they can represent a characteristic element of the presence of cracks.

\section{CONCLUSION AND PROSPECTS}

In this paper, we proposed a flexible prototype for surveying tunnels using two high resolution laser sensors and a stacker embedded on truck. Its deployment is easy, with a quick on-site installation. We were able to acquire a complete tunnel tube $(1.5 \mathrm{~km})$ with a spatial resolution of $1 \times 2 \mathrm{~mm}$, in a few hours, through several passages.

We have shown how we process this data for a $2 \mathrm{D}$ or $3 \mathrm{D}$ visualization highlighting the elements present in tunnels (defects, equipment, etc.). This work is still ongoing and there are many possible improvements. It should be remembered that the LCMS sensors were designed to be used for pavement inspection. Their implementation in a much more complex environment, both geometrically and photometrically, is therefore not straightforward with regard to image visualization and analysis. 

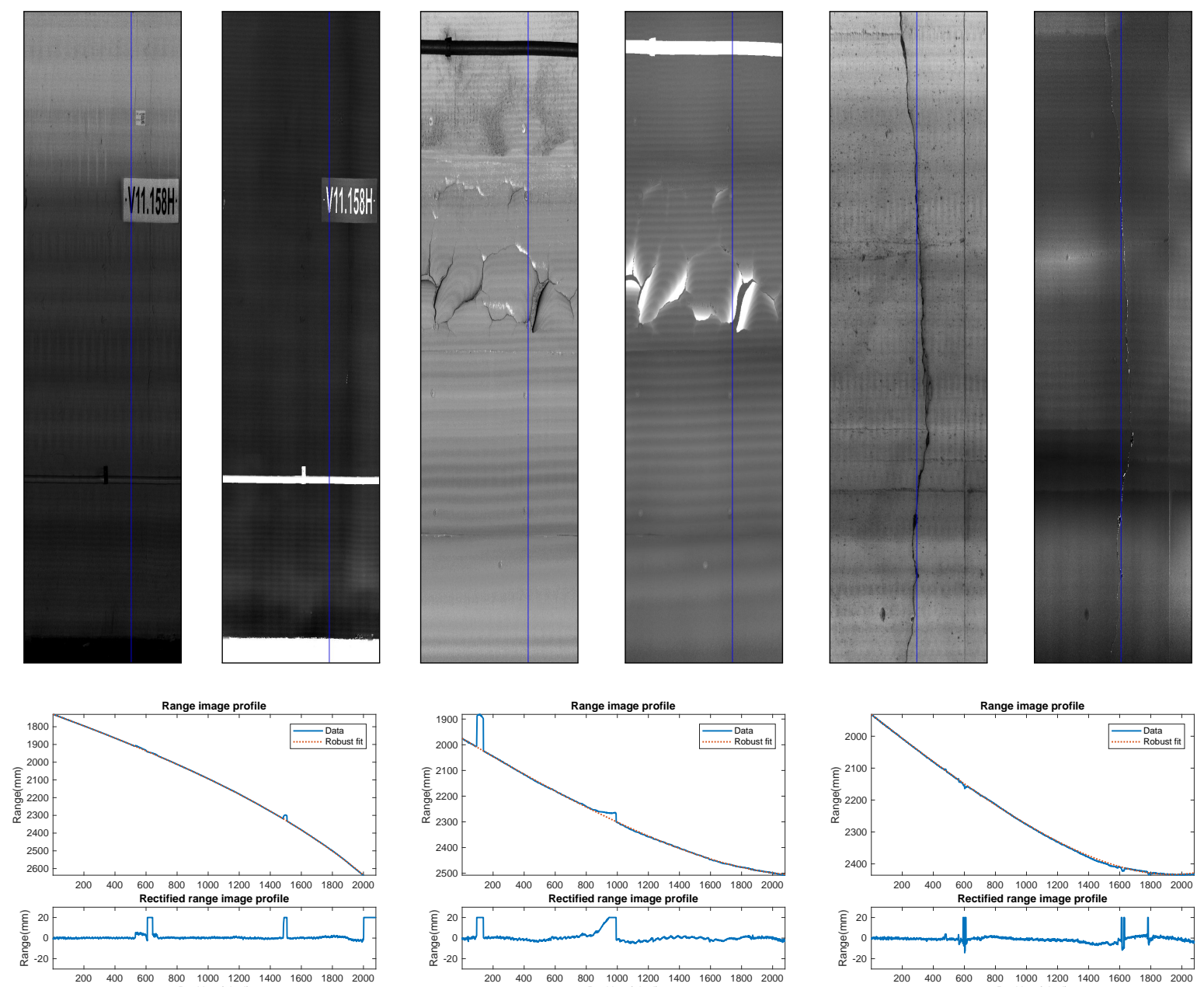

Figure 14. Top: images of range (left) and intensity (right) for 3 different acquisitions. Bottom: range profiles extracted along the column shown in blue on the images, as well as the associated rectified range profile.

The use of the sensors at low speeds results in greater drift of IMUs and the absence of a GPS signal would require the use of more frequent localized landmarks than in the open air to be able to reconstruct an accurate 3D model. Nevertheless, the preliminary results obtained highlight the interest of the joint use of high-resolution 3D information and visual information for the automatic detection of defects and equipment.

The data acquired during these campaigns are currently being prepared for use in learning deep neural networks dedicated to defect detection, that we have already experimented with classic RGB images (Decor et al., 2019). The application of such learning methods requires a fairly long process of image annotation. This must be done on a large number of images, by an expert operator. Thus, even if some visual improvement treatments would not be necessary for learning per se, it is still important to implement them, in an interactive setting, to facilitate the work of the expert. Beyond these technical difficulties, the application of these methods poses other challenges, such as the rarity of certain defects or the generalisation of a learned model from one tunnel to another, for example. However, they offer the advantage of being generalizable to many types of defects, without the need for additional algorithmic development. Moreover, the first results obtained, without the use of depth, are already very encouraging (Decor et al., 2019). Finally, we can consider training a neural network to recognize not only defects, but also equipment.

\section{ACKNOWLEDGEMENTS}

The authors are grateful to the managers and employees of the DIR Normandie and DIR Ile-de-France for making the tunnels available and for their assistance in carrying out the acquisitions. The authors would like to thank Pavemetrics for the adaptation and loan of the sensors and all the staff of the Cerema/CECP in Rouen who developed and built the MALT prototype. They would also like to thank the members of the Cerema/ENDSUM team who carried out the acquisition campaigns with them: Guillaume G, Sébastien, Guillaume D, Christophe, Aude, Lionel and Chris.

\section{REFERENCES}

Besl, P., McKay, N. D., 1992. A method for registration of 3D shapes. IEEE Transactions on Pattern Analysis and Machine Intelligence, 14(2), 239-256.

Cerema, 2019. L'innovation AIGLE 3D : un outil au service d'une gestion patrimoniale intégrée. Accessed on 2019-10-28. https://www.cerema.fr/fr/actualites/accompagnercollectivites-gestion-patrimoniale-innovante. In French.

Decor, G., Bah, M.-D., Foucher, P., Charbonnier, P., Heitz, F., 2019. Defect detection in tunnel images using random forests 
and deep learning. Proceedings of $10^{\text {th }}$ International Conference on Pattern Recognition Systems (ICPRS), Tours, France.

Foroosh, H., Zerubia, J., Berthod, M., 2002. Extension of phase correlation to subpixel registration. IEEE transactions on image processing, 11, 188-200.

Gavilan, M., Sanchez, F., Ramos, J.-A., Marcos, O., 2013. Mobile inspection system for high resolution assessment of tunnel. Proceedings of the 6th international conference on structural health monitoring of intelligent infrastructure, Hong Kong.

Huber, P., 1981. Robust statistics. John Wiley and Sons, New York.

Laurent, J., Fox-Ivey, R., Dominguez, F. S., Garcia, J. R., 2014. Use of 3D scanning technology for automated inspection of tunnels. Proceedings of the world tunnel congress, Foz de Iguazu, Brazil.

Loupos, K., Amditis, A., Stentoumis, C., Chrobocinski, J., Victores, J., Wietek, M., Panetos, P., Roncaglia, A., Camarinopoulos, S., Kalidromitis, V., Bairaktaris, D., Komodakis, N.,
Lopez, R., 2014. Robotic intelligent vision and control for tunnel inspection and evaluation. IEEE International symposium in robotic and sensors environment, Timisoara, Romania, 72-77.

Mohan, A., Poobal, S., 2018. Crack detection using image processing: A critical review and analysis. Alexandria Engineering Journal, 57(2), 787 - 798.

Radopoulou, S.-C., Brilakis, I., 2016. Automated Detection of Multiple Pavement Defects. Journal of Computing in Civil Engineering, 31, 04016057.

Szeliski, R., 2010. Computer Vision: Algorithms and Applications. 1st edn, Springer-Verlag, Berlin, Heidelberg.

Tarel, J., Ieng, S., Charbonnier, P., 2002. Using robust estimation algorithms for tracking explicit curves. Springer (ed.), 6th European Conference on Computer Vision (ECCV), Lecture Notes in Computer Science, 2350, Copenhague, Danemark, 492-407. 